\title{
Science journalists come of age in Pisces
}

\author{
John Gribbin*
}

\section{It seems that more science journalists are born under Pisces than any other sign.}

WINDSOR $^{1}$ has pointed out that the astrological belief in the importance of the 'Sun sign' as an influence on personality seems to be reflected in the distribution of birth dates of American biologists. In particular, he found that molecular biologists tend to be born under Aries. I have now compared the birthdays of science journalists found in the London office of Nature with those of all staff members found in that office. The birthdays were obtained by personal questioning during August, 1974, and the appropriate Sun signs were assigned.

More science journalists were born under the sign of Pisces than any other sign (Fig. 1), and relatively few (zero) members of the Nature staff were born under Aries, Taurus or Gemini. In spite of the small size of the sample, the result is significant as indicated by Lynden-Bell's half-power test. This test uses the criterion that in statistics, all numbers go as the square root (D. Lynden-Bell, unpublished).

Taking the data for science journalists alone, there are eight Pisces and eight others (including one "no comment"). The most densely populated Sun sign other than Pisces is Leo, with just two members-less than even the half-power of the Pisces population.

It may also be significant that of the Leo population one (E) is the Editor of Nature and the other (DE) is Deputy Editor.

* Nature, London

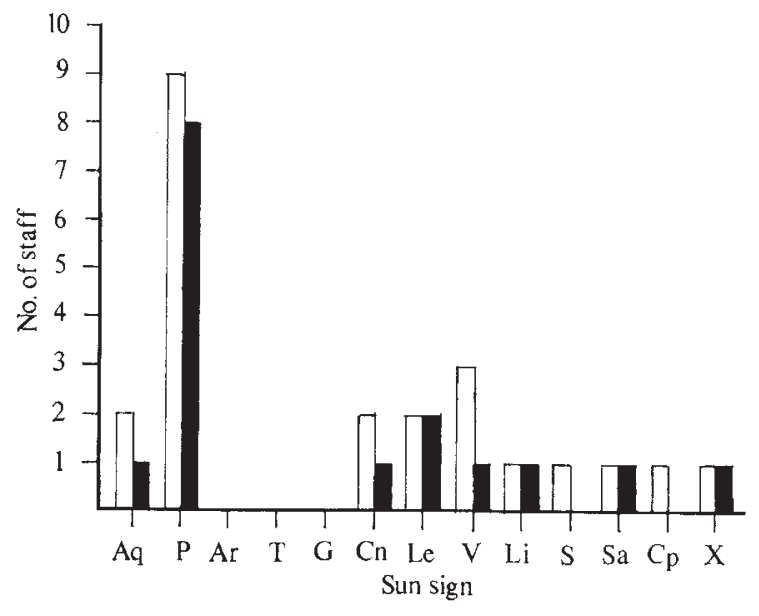

Fig. 1 Frequencies of Sun signs of Nature staff. Open histogram, all staff; solid histogram, science journalists only; $\mathrm{X}$, birthday not revealed ("no comment").

While these results are suggestive, further observations of a larger sample are needed before any firm conclusions can be drawn.

1 Windsor, D. A., Nature, 248, 788 (1974).

\section{Ambisonic reproduction of directionality in surround-sound systems}

\author{
P. B. Fellgett*
}

In both the technology and the aesthetics of extending high fidelity reproduction to surround-sound, reproduction of natural ambience is crucial. The 'quadraphonic' attempt to reproduce four stereoblended tracks, derived from multi-microphone mix-down, cannot provide this. Complete spherical directionality can however be encoded on to a minimum of two audio channels to produce acoustically acceptable surround-sound systems. Limitations are set both by the number of available loudspeakers and by the number of channels.

THE history of representational art has many examples of what people of a particular age and culture were unable to perceive, and therefore to reproduce; for instance the inability of the dynastic Egyptians to perceive that the full-face eye is not seen in a profile figure. The reproduction of sound follows a similar pattern. In the early development of the phonograph and of 'wireless' the listener was so satisfied with the novelty of hearing the human voice or music issue from a machine that he demanded nothing else. Indeed there was often little conscious

* Address: Department of Engineering and Cybernetics, University of Reading, Whiteknights, Reading RGL 2AL. appreciation that anything was lacking in fidelity of reproduction; how else could Sir Arthur Conan Doyle suppose in The Adventure of the Mazarin Stone that Sherlock Holmes could deceive a sophisticated villain into mistaking a 'modern gramophone' of 1927 for real violin playing, even in the next room. As late as the 1930 s, many listeners could see little short of perfection in the commercial 'radiogram'.

As the sense of wonder wore off, however, a more critical attitude filtered down from audio engineers to the buying public, and the search began for high fidelity reproduction.

During and immediately after the Second World War, domestic reproduction of sound depended on the 78-r.p.m. 